\title{
The residency application process: Challenges for our specialty
}

\author{
Azad S. Karim, MD, and Shari L. Meyerson, MD
}

\footnotetext{
From the Division of Thoracic Surgery, Northwestern University, Feinberg School of Medicine, Chicago, Ill. Received for publication Aug 13, 2016; revisions received Nov 1, 2016; accepted for publication Nov 7, 2016; available ahead of print March 14, 2017.

Address for reprints: Shari L. Meyerson, MD, Division of Thoracic Surgery, Northwestern University Feinberg School of Medicine, 676 N. Saint Clair St, Suite 650, Chicago, IL 60611 (E-mail: smeyerso@nm.org).

J Thorac Cardiovasc Surg 2017;153:1618-20 $0022-5223 / \$ 36.00$

Copyright (C) 2017 by The American Association for Thoracic Surgery http://dx.doi.org/10.1016/j.jtcvs.2016.11.073
}

Feature Editor's Note-As highlighted in the original New England Journal of Medicine article published in early 2016 by Aagaard and Abaza titled "The Residency Application Process: Burden and Consequences," ${ }^{\prime}$ both resident applicants and program directors make a tremendous investment of time and money to match positions for graduate medical education. This comes at the price of making the fourth year of medical school increasingly dedicated to securing such graduate medical education positions rather than learning. For the resident, the process usually involves completing multiple applications, securing subinternship "audition" rotations, and traveling plus interviewing at multiple programs. For the training programs, this usually means interviewing 30 to 40 almostindistinguishable applicants for 1 or 2 integrated positions. In cardiothoracic surgery, because we have more than one available training track (integrated and traditional), this forces the applicants to hedge bets and apply to both integrated thoracic residencies as well as traditional general surgery residencies, potentially compounding the application problem.

Karim and Meyerson have described on how this plays out in our specialty by surveying both program directors and recent integrated applicants for 2016. Multiple suggestions have been proposed for streamlining the application process and should be considered in further detail; such an effort may be best led by the Thoracic Surgery Directors Association: regionalization of the interview process, more detailed program descriptions and priorities, better coordination between programs interview dates, and meaningful ongoing discussions between thoracic and general surgery program directors to optimize the training opportunities for outstanding cardiothoracic applicants. The second ancillary issue is how to better prepare residents educationally for our cardiothoracic surgical residency. Since cardiothoracic surgery has developed its own electronic content and learning

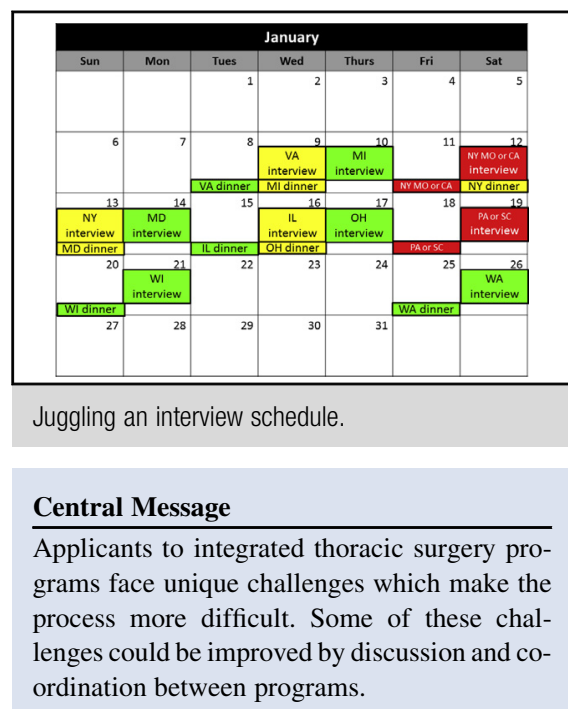

See Editorial Commentary page 1621. management system (Thoracic Surgery Curriculum) and historically run a simulation-based, early integrated resident national boot camp, this may be an opportunity for us as a specialty to creatively develop a prerequisite curriculum or implement a "preresidency" boot camp to enhance preparation for residency during the second half of the fourth year of medical school.

\section{Edward Verrier, $M D$}

Aagard and Abaza, ${ }^{1}$ in their recent article in the New England Journal of Medicine, highlighted the burden, on both residents and programs, as well as the missed opportunities for education generated by the current residency application process. They noted that a fourth-year medical student, on average, will begin the year with 1.8 months of away or audition rotations, submit 36 applications, and travel for 12 interviews, all leading up to match day. The remainder of the fourth year often is used for vacation or travel, with less than $50 \%$ of students doing any clinical work after match day. With the limited time spent on clinical learning, the fourth year ultimately falls short of preparing students for their chosen internship and residency. Aagard and Abaza also highlighted the challenges for program directors, who have difficulty sifting through the overwhelming number of applications received. ${ }^{1}$ Applicants know the match is competitive and feel pressured to apply to more and more programs. This 
creates a spiraling increase in application numbers-more than a program director can hope to read.

To gain insight into the impact the match process has on our specialty of thoracic surgery, we surveyed all program directors and the current national integrated thoracic surgery intern class. Responses were received from $45 \%$ $(11 / 24)$ of programs and $38 \%$ (14/37) of the current interns. The survey was completely anonymous and consisted of 6 multiple choice questions concerning the number of applications submitted and received, interviews offered and attended, and requested suggestions to improve the process in a final open response question.

From the program director perspective, the process seems much less overwhelming in a small specialty like thoracic surgery. We are blessed with a strong applicant pool of a manageable size. Sixty percent of responding programs received applications from nearly all of the 103 applicants in the pool in 2016. Compared with a general surgery pool of 2345 applicants, ${ }^{2}$ it is reasonable to read and review each of the thoracic surgery applications in detail whereas larger specialties often use objective cut points such as United States Medical Licensing Examination scores to decide which applications to read. With only 1 to 2 positions per program, most programs interviewed 20 to 30 applicants over 1 to 2 days, creating limited interruption to the normal clinical flow.

Based on multiple discussions with both previous and current applicants, however, the application process becomes even more overwhelming if they wish to pursue a highly competitive specialty like thoracic surgery. In the 2016 general surgery match, there were 1.89 applicants per position compared with thoracic surgery with 2.78 applicants per position. ${ }^{2}$ Many applicants feel the need to buffer their list with general surgery programs as well, doubling their workload and complicating their schedules. Although thoracic surgery programs clearly understand the need to have a backup plan, general surgery programs are perceived to be biased against applicants to integrated programs, feeling they are not committed to general surgery. Applicants feel the need complete 2 separate applications, adjusting their general surgery personal statement and letters to deemphasize their interest in thoracic surgery. They are afraid to apply to both programs at the same institution out of concern that the general surgery program will "find out" they are an integrated thoracic applicant and not give them fair consideration. It is unclear how much of this fear is grounded in reality, but the concern is likely valid in at least some institutions. General surgery program directors of course seek to match only the best applicants; however, their definition of best may include only those who want to be general surgeons. These are important discussions in which thoracic surgeons should be intimately involved at their institutions. It is important to encourage general surgery program directors to remember that a thoracic surgeon trained through a traditional pathway is not a "waste" of their residency slot and in fact they will gain an excellent resident who will go on to be a credit to their training program. Because only $36 \%$ of applicants will match in thoracic surgery, general surgery programs will miss out on excellent applicants who are likely to be successful in a general surgery residency and hopefully will proceed to thoracic surgery through the traditional pathway. We need to help them recognize that the thoracic surgeon is not wasting their general surgery training any more than the breast surgeon or the hepatobiliary surgeon who never does another colon resection or appendectomy after completing general surgery.

Interview dates present another unique challenge. Thoracic surgery applicants feel the need to interview at more programs than their colleagues in less-competitive specialties. The majority interviewed at 11 to 15 thoracic programs and then added up to 10 general surgery interviews, creating a significant travel and financial burden. Despite that burden, the primary concern of our current interns about the interview process was conflicting interview days. With such a small pool of programs, it is difficult for applicants to choose between 2 interviews offered on the same day. This issue is even more important for those who may not be at the top of the applicant pool, as they are trying to balance a larger number of general surgery interviews as well. One possible solution to address this issue would be to have one central calendar for all programs with interview dates. Perhaps assigning blocks of interview time to regions of the country would help as well so applicants could schedule a single East Coast or West Coast trip rather than flying back and forth across the country. This would improve transparency in the process and avoid interview overlap, benefitting both the applicants and the programs.

Even with these challenges, 37 applicants matched into thoracic surgery last year. ${ }^{2}$ After match day, however, the majority of matched applicants remained suboptimally prepared for their internship. Aagaard and Abaza ${ }^{1}$ suggested developing milestones for students that would improve preparedness tailored to the field they have chosen. For students matched in thoracic surgery, this might include elective rotations in cardiology, pulmonary medicine, or radiology to increase knowledge base and experience with patients, diagnostic tests, and procedures relevant to succeeding as a thoracic surgery intern. Challenging rotations after match day also will serve to refresh clinical skills that may become rusty or lost during the long hiatus of interview season.

In conclusion, residency applicants and incoming interns face various challenges unique to thoracic surgery that are important to identify and address. Potential ways to address these challenges include specifically communicating with general surgery programs at our institutions to encourage them to give integrated thoracic applicants an unbiased review. Coordinating interview schedules by creating a transparent national calendar/structure also would decrease the 
stress level for our applicants. Setting program-specific recommendations for fourth-year student rotations to maximize preparation for thoracic surgery training for candidates who have successfully matched into an I6 position could provide a roadmap for students to better use their final months of medical school benefitting programs, interns, and the patients we all serve.

\section{Conflict of Interest Statement}

Authors have nothing to disclose with regard to commercial support.
The authors thank the Thoracic Surgery Directors Association for their support of and assistance with our survey of programs and interns.

\section{References}

1. Aagaard EM, Abaza M. The residency application process-burden and consequences. N Engl J Med. 2016;374:303-5.

2. American Association of Medical Colleges. 2016 Main Residency Match data. Available at: http://www.nrmp.org/match-data/main-residency-match-data/?utm source $=2016+$ May + The + Match + Illuminator + \&utm_campaign $=$ May $+2016+$ Illuminator\&utm_medium=email. Accessed August 8, 2016. 